\title{
16. PHYSICAL STUDY OF PLANETS AND SATELLITES (ÉTUDE PHYSIQUE DES PLANÈTES ET SATELLITES)
}

\author{
PResident: G. H. Pettengill. \\ VICE-PREsident: S. K. Runcorn. \\ Organizing Committee: J. Connes, S. Miyamoto, V. I. Moroz, T. C. Owen, B. A. Smith.
}

\section{INTRODUCTION}

The last three years have been marked by significant progress in the use of space probes to study Venus and Mars, and the new results from these have tended to dominate the field of our Commission's interest. The arrival of the U.S.S.R. orbiters, MARS 2 and 3, and the U.S. orbiter, Mariner 9, at Mars in the late summer of 1971 initiated a long period of intensive, close-up observation of that planet which has begun to equal that previously available only for our nearest celestial neighbor, the Moon. More recently, new information has been obtained on the characteristics of the dense atmosphere of Venus through the observations relayed back by the U.S.S.R. probe, Venera 8, in July 1972. While they have tended to be obscured by the more spectacular space missions, groundbased observations have also made solid progress in providing new data on the planets since the Brighton meeting in 1970.

In keeping with the tradition established by J.S. Hall in the last triennial report and with the intent of brevity expressed by the IAU Executive Committee, this report will contain only short summaries of recent research and no comprehensive bibliography. A symposium on Planetary Atmospheres and Surfaces, jointly sponsored by the IAU, URSI and COSPAR, was held May 1972 in Madrid, and presented 52 papers touching on the chief scientific results obtained in this field in the last three years. The papers presented at this symposium are published in 4 successive issues of Icarus: Vol. 17, Nos. 2 and 3 (1972) and 18, Nos. 1 and 2 (1973).

This report contains short summaries of progress in various aspects of planetary research followed by some notes of ongoing unpublished research at various centers, a report on the Planetary Data and Research Centers at Meudon and Flagstaff, a note on the International Planetary Patrol Program and a brief statement from the Working Group on Martian Nomenclature.

RESEARCH IN PLANETARY ATMOSPHERES, 1970-1972

D. M. Hunten

\section{General comments}

During the period covered here, two major review papers have appeared: 'The Atmospheres of Mars and Venus' by A. P. Ingersoll and C. B. Leovy, Ann. Rev. Astron. Ap. 9, 147 (1971); and 'Composition and Structure of Planetary Atmospheres' by D. M. Hunten, Space Sci. Rev. 12, 539 (1971). IAU Symposium 40 has been published, and papers from several of the Arizona conferences have appeared in the Journal of Atmospheric Sciences. In press as this is written, in Space Science Reviews, is 'A Survey of the Outer Planets, Jupiter, Saturn, Uranus, Neptune, Pluto, and their Satellites' by R. L. Newborn, Jr. and S. Gulkis.

\section{Mercury}

J. R. Gott and A. E. Potter have modelled the production of a transient atmosphere produced by neutralization of the solar wind. $H$ concentrations near the subsolar point are predicted to be $2700 \mathrm{~cm}^{-3}$. 


\section{Venus}

Two more landers, Venera 7 and 8, were sent to Venus, and succeeded in confirming directly the surface temperature of $750 \mathrm{~K}$, from which a pressure of 90 bars was inferred. According to early reports from Venera 8 , some solar radiation may penetrate to the surface, and free $\mathrm{NH}_{3}$ is present in the atmosphere.

The $\mathrm{L} \alpha$ data from Mariner 5 had led to suggestions that the planetary corona contains an important component of deuterium. L. Wallace tested this hypothesis with a rocket-borne spectrometer that resolved the isotopic structure of $\mathrm{L} \alpha$, but failed to observe any component due to D. H. W. Moos and G. J. Rottman observed a strong atomic-oxygen dayglow in the $\lambda 1304$ triplet and the $\lambda 1356$ forbidden line but an analysis by D. J. Strickland is unable to account for the observed strength or ratio. Nevertheless, there is a strong suggestion that upper-atmospheric oxygen may be more abundant on Venus than on Mars.

Polarimetry by D. L. Coffeen and T. Gehrels has been interpreted by J. E. Hansen to give information about the uppermost (i.e. visible) layer of the Venus clouds. The index of refraction in the visible is $1.44 \pm 0.01$, the mean particle diameter is $2.2 \mu$, and the particles are spherical. Further observations have been published by A. Dollfus and Coffeen. J. S. Lewis has proposed that the composition is very concentrated aqueous $\mathrm{HCl}$; B. Hapke has made a similar suggestion, but added a trace of iron, which helps raise the refractive index and gives a plausible coloration. An earlier suggestion of hydrated ferrous chloride crystals has been examined by D. P. Cruikshank and A. B. Thomson who find the spectral evidence inconclusive. It may also be noted that crystals of any kind are incompatible with the polarization data.

A large number of $\mathrm{CO}_{2}$ spectra by L. D. G. Young and R. A. Schorn have been examined by G. E. Hunt in the framework of a multilayer radiative-transfer model. He prefers a cloud structure consisting of a dense layer at $100-200 \mathrm{mb}$, with a thin haze (or a thin layer) far above it. A similar structure is obtained by W. A. Traub and N. P. Carleton, who apply the same kind of model to interpret the detailed line profiles. J. L. Regas and others, studying models with only a single cloudy region, prefer cloud-top pressures around $10-100 \mathrm{mb}$. They agree with earlier results in rejecting ice as a probable constituent since the $\mathrm{H}_{2} \mathrm{O}$ lines are far too weak. A number of other workers including J. W. Chamberlain, M. J. S. Belton, W. M. Irvine, D. J. Van Blerkom, and M. Kanal, are examining basic radiative-transfer problems in a more general way.

A number of investigations of the radio spectrum have appeared. J. B. Pollack and D. Morrison have studied the brightness-temperature transition between $0.2 \mathrm{~mm}$ and $10 \mathrm{~cm}$, and find evidence for about $0.5 \% \mathrm{H}_{2} \mathrm{O}$ below the cloud tops, while an interferometer study by A. C. E. Sinclair $e t$ al. at $11 \mathrm{~cm}$ finds $0.65 \%$. The inferred absorption is not specific to $\mathrm{H}_{2} \mathrm{O}$, however, and contributions from other substances are possible. Sinclair et al. find a remarkably uniform surface temperature, with the poles $16 \mathrm{~K}$ warmer than the equator. They do not confirm the $50 \mathrm{~K}$ hot spot reported by R. W. Hall and N. J. B. A. Branson at $6 \mathrm{~cm}$.

The comparative absence of water has been discussed by a number of people. J. S. Lewis's models of condensation from the solar nebula suggest that water may never have accumulated on Venus in the first place. On the other hand, an original terrestrial abundance might well have disappeared as a consequence of the larger solar flux at Venus. The 'runaway greenhouse effect' has been modelled by A. P. Ingersoll, S. I. Rasool, C. de Bergh, and J. B. Pollack. All agree that a Venus ocean would probably evaporate completely; photolysis and hydrogen escape could then remove a huge amount of water. The latter processes have been discussed by L. L. Smith and S. H. Gross. Pollack points out that the reduced solar luminosity early in the age of the solar system might have permitted oceans to survive for some time before the greenhouse effect 'ran away'.

\section{Mars}

The study of the atmosphere of Mars has been dominated by the flybys of Mariners 6 and 7, and the successful orbiter, Mariner 9 (Science 175, 293-329, 1972). Nevertheless, Earth-based spectroscopy has had its own triumph: the forty-year search for $\mathrm{O}_{2}$ has apparently finally succeeded. 
W. A. Traub and N. P. Carleton, and independently E. S. Barker, find mixing ratios in the neighborhood of $0.08 \%$. Not long after these observations, the ultraviolet spectrometer of C. A. Barth aboard Mariner 9 detected ozone, in clear association with the polar hood and cap. This molecule cannot be detected at lower latitudes. R. A. Hanel's infrared spectrometer observed $\mathrm{H}_{2} \mathrm{O}$, in amounts of 10-20 $\mu$ precipitable; concurrent observations from Earth are in agreement. Apparently the amounts were typically less in 1971 than in 1969.

A vast amount of information has been obtained on haze, clouds, and dust. The 1971 perihelic opposition saw a planet-wide dust storm which was in full swing as Mariner 9 arrived. In fact, it was only after several months that clear photographs of the surface were obtained. Many other kinds of cloud phenomena were photographed later in the mission. A preview of many of the results was obtained by Mariners 6 and 7 (C. B. Leovy et al., J. Geophys. Res. 76, 297, 1971).

Temperature profiles were obtained from occultation of the orbiter's radio signals and by inversion of the $\mathrm{CO}_{2}$ band profiles from the on-board infrared spectrometer. During the dust storm, the Martian stratosphere was found to be remarkably warm (as high as $220 \mathrm{~K}$ ). Later in the Mariner 9 mission, this temperature fell to $180 \mathrm{~K}$; the even lower temperatures inferred from Mariner 6 and 7 data were not confirmed by the infrared experiment. The frequent occurrence of high haze layers at the limb, however, suggests temperatures below the frost point of $\mathrm{CO}_{2}$.

The upper atmosphere, studied by radio occultation and ultraviolet spectroscopy, shows an exospheric temperature of $350 \mathrm{~K}$. The generally lower electron density observed in 1971 reflects the drop of solar activity since 1969, but the absolute value is greater than predicted by about a factor of two. The observed $\mathrm{L} \alpha$ intensities lead to a flux of escaping hydrogen of $2 \times 10^{8} \mathrm{~cm}^{-2} \mathrm{~s}^{-1}$, which seems remarkably stable; for example, the dust storm had no effect. The photodissociation of the observed water can account for the observed hydrogen flux, according to D. M. Hunten and M. B. McElroy. In their theory, molecular hydrogen forms an important intermediate and provides a reservoir to stabilize the rate of escape.

D. J. Strickland, G. E. Thomas, and P. R. Sparks have discussed in great detail the measurements of the OI resonance-triplet from Mariners 6 and 7. They conclude that the mixing ratio of atomic oxygen is 0.5 to $1.0 \%$ at the ionospheric maximum $(135 \mathrm{~km})$.

Surface topography has been measured by several independent methods which give either true height (occultation points, Earth-based radar) or pressure height (occultation, infrared $\mathrm{CO}_{2}$ absorption, ultraviolet scattering). The $\mathrm{CO}_{2}$-absorption method has also been applied from the Earth but with substantially reduced resolution. The total height range noted, about $20 \mathrm{~km}$, was nearly the same for all methods. There were important differences between the pressure and geometric heights in certain areas (notably the Tharsus region), however, which are attributed to substantial, but local, gravitational anomalies.

A number of papers have discussed dynamical models of the Martian atmosphere. For several years the best description of the vertical structure near the surface has been thought to be the convective model of $P$. Gierasch and $R$. Goody. However, observed lapse rates have always tended to be less than the adiabatic value predicted there. This tendency was very marked during the Martian dust storm, but not confined to that period. P. Stone has presented a simplified model of the baroclinic instability which seems to agree much better with the observations. Other work has stressed the importance of local winds, particularly with a view to explaining how dust is raised from the surface. S. Blumsack has discussed the effect of a large protrusion or basin, pointing out that these will produce permanent thermal anomalies with associated winds. P. Gierasch, C. Sagan, and J. Veverka have applied these ideas, and sought specific regions on Mars where strong winds would be expected. Gierasch and Goody have discussed the local solar heating to be expected in a dust cloud, and pointed out that a 'dust hurricane' should be expected if the conditions are right. They suggest this mechanism as the initial source of the planetwide dust storm observed in 1971 and earlier perihelic oppositions.

Aeronomical topics have also received a good deal of study. $\mathrm{CO}$ and $\mathrm{O}$ are surprisingly rare in the upper atmosphere, despite the rapid photolysis of $\mathrm{CO}_{2}$. It has been necessary to assume very efficient downward transport, either by strong eddy mixing or an even stronger large-scale circula- 
tion. Alternatives have been suggested, but have not survived careful examination (e.g. M. B. McElroy and J. C. McConnell, J. Atmos. Sci. 28, 879, 1971, and other papers in that issue). Once out of the upper atmosphere, the dissociation products have still been an embarrassment, because $\mathrm{O}$ tends to form $\mathrm{O}_{2}$ instead of reacting with $\mathrm{CO}$. It has seemed likely for some time that the explanation lies in catalysis by the photo-dissociation products of $\mathrm{H}_{2} \mathrm{O}$ ('odd hydrogen', $\mathrm{H}, \mathrm{OH}$, and $\mathrm{HO}_{2}$ ). Two detailed models of such an effect have recently been published. That of McElroy and T. M. Donahue prevents the buildop of too much $\mathrm{O}_{2}$ by rapid downward mixing through the stratosphere, while oxidation of $\mathrm{CO}$ by $\mathrm{OH}$ then restores the balance. T. D. Parkinson and D. M. Hunten permit the $\mathrm{O}_{2}$ to form, but postulate conditions favorable to the production of $\mathrm{H}_{2} \mathrm{O}_{2}$. They also address the production of ozone, which is rather efficiently destroyed by odd hydrogen. The lack of ozone at low latitudes is readily explained in this way; its presence in cold regions is presumably due to the suppression of odd hydrogen. The freezing out of $\mathrm{H}_{2} \mathrm{O}$ presumably contributes to this suppression, but does not seem to yield a complete explanation, because $\mathrm{H}_{2}$ is also an important source of odd hydrogen.

Several groups have published lists of upper limits for minor constituents. These are D. Horn, G. C. Pimentel, and others (Mariner 6 and 7 infrared experiment); R. Beer et al. (Earth-based infrared); and T. Owen and C. Sagan (OAO-2 Ultraviolet spectrometer). The last of these also discusses Jupiter, Saturn, and Venus.

\section{The Jupiter system}

No less than two minor isotopes have been observed on Jupiter. R. Beer and F. W. Taylor have analyzed a measurement of $\mathrm{CH}_{3} \mathrm{D}$ and obtained a $\mathrm{D} / \mathrm{H}$ ratio significantly less than for Earth (a factor of $\frac{1}{2}$ to $\frac{1}{6}$ ), while $\mathrm{K}$. Fox and others have found $\mathrm{C}^{13} \mathrm{H}_{4}$ in an abundance consistent with the terrestrial value.

Our concepts of the structure of Jupiter have been overturned by the excess infrared emission observed by H. H. Aumann, C. M. Gillespie, and F. J. Low. The implication is that internal heat is carried up by deep convection, and that most of the interior is well stirred. Two other lines of evidence have now been presented. R. L. Wildey and L. M. Trafton have observed and analyzed the limb darkening in the 8-14 $\mu$ band. The effective temperature is found to be $135-140 \mathrm{~K}$, in excellent agreement with direct bolometry. S. Gulkis and R. Poynter have analyzed the microwave thermal emission spectrum and found that the temperature gradient is adiabatic to considerable depths. They also derive an abundance for $\mathrm{NH}_{3}$ in good agreement with the solar value.

At $5 \mu$, where the atmospheric gases are fairly transparent, the major opacity is normally due to clouds. J. A. Westphal has observed several 'hot spots', with temperatures of at least $310 \mathrm{~K}$. The implication is that he is seeing through holes in the clouds to much greater depths than normal.

The Danielson-Tomasko model of line formation in the Jupiter clouds has been extended by $L$. Axel. In addition to the lower cloud deck and an upper layer with an optical thickness of 3.5 , he suggests a still higher haze of small absorbing particles. This haze explains the low ultraviolet albedo, and has important potential effects on the stratospheric heat balance. Other work on line formation has been done by G. E. Hunt and J. A. Margolis.

An occultation of $\beta$ Sco gave opportunity for measurements of the stratospheric scale height. Several expeditions from the University of Texas (W. B. Hubbard et al.) obtained a mean value of $29 \mathrm{~km}$, much closer to expectation than the 20 -yr value of $8 \mathrm{~km}$. A serious difficulty is the determination of the zero level in the presence of the light from the planet. Perhaps this explains the $8 \mathrm{~km}$ found from $\beta$ Sco by K. C. Freeman and N. R. Stokes, who were forced by bad seeing to use a very large diaphragm. M. Combes, J. Lecacheux, and L. Vapillon have given a preliminary scale height of $30 \mathrm{~km}$ for great altitudes.

Satellite eclipses have been studied by M. J. Price and others. The conclusion is that the atmosphere is more opaque than expected at the $100-\mathrm{mb}$ level. They suggest the presence of aerosols.

Narrowband photometry of 4 regions of Jupiter has been accomplished by C. B. Pilcher et al. The continuum albedos are found to be considerably larger than previous values. These low values had caused difficulties in the interpretation of spectral line formation in the scattering atmosphere. 
The aeronomy of methane has been studied by D. F. Strobel. He finds methane itself to be the major hydrocarbon at all heights, but that acetylene is probably the most important infrared radiator at the mesopause. He also finds that ionospheric heating is not very effective: the temperature rise across the thermosphere is only $10-20 \mathrm{~K}$. M. Shimizu has also studied this problem.

L. Wallace and D. M. Hunten have studied the $\mathrm{L} \alpha$ albedo, which is due to $\mathrm{H}$ in the thermosphere and has been measured by $\mathrm{H}$. W. Moos and G. J. Rottman. The amount of $\mathrm{H}$ is surprisingly large, and difficult to attain in atmospheric models unless the mixing rate is much less than at comparable heights on Earth. Models of the ionosphere have been computed by S. S. Prasad and L. A. Capone. The ion densities cover a large range of uncertainty because of our ignorance of some important reaction rates.

One component of $\beta$ Sco was also occulted by Io, and several expeditions attempted to get observations. The best data were obtained by B. A. Smith, who derive a probable limit of $0.1 \mu$ bar to the surface pressure. Such an atmosphere can still readily support an ionosphere, but probably not the frost formation during eclipse reported some time ago by A. B. Binder and D. P. Cruikshank. Attempts to repeat this observation, by several observers on several occasions, have given ambiguous results. Similar observations of Europa have not shown the post-eclipse brightening. Formation and conductivity of Io's ionosphere are discussed by D. L. Webster, A. Y. Alksne, and R. C. Whitten.

\section{Saturn and Titan}

New measurements of Saturn's $\mathrm{H}_{2}$ quadrupole lines have been made by L. M. Trafton; they may be slightly weaker than on Jupiter. He has also revised the methane abundance, to about $40 \mathrm{~m}$-atm. Circular polarization in the polar region has been measured by J. B. Swedlund, J. C. Kemp, and R. D. Wolstencroft. The millimeter-wave spectrum, measured by G. T. Wrixon and W. J. Welch, shows good evidence for ammonia.

A great deal of new information has appeared about the atmosphere of Titan, which is turning out to be one of the most interesting in the solar system. Trafton has greatly revised the methane abundance by taking account of the pressure dependence of absorption. A pure $\mathrm{CH}_{4}$ atmosphere has an abundance of $1.6 \mathrm{~km}$-atm; if there is less methane, it must be accompanied by a still larger amount of some other gas. Trafton also presents evidence for $\mathrm{H}_{2}$ in an abundance of $5 \mathrm{~km}$-atm, and discusses the large escape rates thus implied. In addition, he finds diffuse absorptions that are also present on Saturn and Uranus.

Thermal emission in the 8-20 $\mu$ region has been measured by F. C. Gillett and W. Forrest; D. A. Allen and T. L. Murdock; and D. Morrison, D. P. Cruikshank, and R. E. Murphy. There is a strong implication of a large greenhouse effect, with a temperature of $93 \mathrm{~K}$ at $20 \mu$, rising to $140 \mathrm{~K}$ at wavelengths shorter than $10 \mu$. Several people have proposed pressure-induced opacity in $\mathrm{H}_{2}$ as responsible. Morrison $e t$ al. and C. Sagan and G. Mullen, suggest a large amount of pure $\mathbf{H}_{2}$, but such an atmosphere cannot be gravitationally bound by Titan. J. B. Pollack, and J. S. Lewis and R. G. Prinn, use an $\mathrm{H}_{2}-\mathrm{CH}_{4}$ mixture, and D. M. Hunten an $\mathrm{H}_{2}-\mathrm{N}_{2}$ mixture. In either case, the heavy gas can in principle stabilize the atmosphere against blowoff, though there is still a large escape flux of $\mathrm{H}_{2}$. J. Veverka has presented evidence for clouds, based on polarization measurements.

Ideas about the atmospheric composition of Titan have been considerably influenced by $\mathbf{J}$. S. Lewis's model of condensation in the solar nebula. The proportions by mass are suggested to be about one-half silicates, one third- $\mathrm{H}_{2} \mathrm{O}, 10 \% \mathrm{NH}_{3}$, and $4 \% \mathrm{CH}_{4}$. Only the last is volatile enough to be present in large amounts in the atmosphere.

\section{Uranus and Neptune}

The microwave spectra of both planets have been measured and briefiy discussed by $\mathrm{C}$. $\mathrm{H}$. Mayer and T. P. McCullough, and also W. J., A. C., and G. T. Webster. As for Jupiter and Saturn, these wavelengths arise from rather deep in the atmosphere and indicate an increase of temperature with 
depth. D. A. Harper, Jr., F. J. Low, G. H. Rieke, and K. R. Armstrong have measured the surprisingly low temperature of $45 \mathrm{~K}$ at $350 \mu$. B. L. Lutz and D. A. Ramsay have identified several new $\mathrm{CH}_{4}$ bands in the $7500 \AA$ region.

A paper by M. J. S. Belton, M. B. McElroy, and M. J. Price has suggested that the $\mathrm{H}_{2}$ quadrupole lines on Uranus are best explained if the atmosphere is completely free of cloud. L. Wallace has investigated this suggestion further, and has calculated the intensity of the Raman 'ghosts' that would be produced by the shifting of Fraunhofer lines to longer wavelengths. For a purely gaseous atmosphere these ghosts should have an intensity about $10 \%$ of the original line. J. S. Lewis and R. G. Prinn have considered the condensation of the expected amount of methane and concluded that cloud should form. R. E. Danielson, M. G. Tomasko, and B. D. Savage have analyzed a sequence of pictures of Uranus from Stratoscope II, with a resolution of 0 "2. No bands or belts can be seen, but the limb-darkening function suggests the presence of cloud. The diameter is found to be $51800 \pm 600 \mathrm{~km}$. J. A. Westphal, and also W. M. Sinton, have found limb brightening at wavelengths inside deep methane bands. Again, the simplest explanation is the presence of a cloud layer below an optically thin scattering atmosphere. But Belton and Price have shown that even this result can be explained in a clear atmosphere with pressure-induced absorption.

Photometry of Uranus has been presented by R. L. Younkin; J. F. Appleby and W. M. Irvine; and A. B. Binder and D. W. McCarthy.

\section{PLANETARY SURFACES}

Much of the expanded interest in planetary surfaces has come about as a result of the spectacular recent results obtained from spacecraft. These observations, particularly in the case of Mars, are beginning to permit geological studies of a planetary surface which are in at least a superficial way similar to those for Earth. It is obviously impossible to treat here the wealth of photographic and other instrumental results obtained by Mariner 9 and Mars 2 and 3. For these, the reader is directed to the Symposium and other papers presented in Icarus 17, No. 2 (Oct. 1972). Papers on Mercury and Venus are contained in the subsequent issue 17, No. 3 (Dec. 1972), while Earth-based observations of Mars will be found in 18, No. 1 (Jan. 1973). Volume 18, No. 2 (Feb. 1973) contains the Symposium (and related) papers on the Giant Planets.

\section{Mercury}

McCord and Adams report that the spectral reflectivity curve for Mercury is very similar to that corresponding to upland and mare regions of the Moon. In the case of the Moon, it is known that the slope of this curve is controlled by titanium- and iron-rich glass in the soil, and these authors suggest that the surface of Mercury is likely also to be rich in glasses colored by these elements. Dollfus has prepared a map of surface features and, with J. B. Murray and B. A. Smith, quotes a measured rotational period for Mercury of $58.644 \pm 0.009$ days, in good agreement with the $\frac{3}{2}$ orbital-synchronous value of $58 \cdot 646$ days.

R. M. Goldstein and D. B. Campbell have obtained radar maps of some scattering features on the surface of Mercury at wavelengths of 12.5 and $70 \mathrm{~cm}$, respectively. The relatively low signal-tonoise ratio prevents these from being completely unambiguous, however, and they are looking forward to more favorable observations. The equatorial topography has been obtained from radar observations by R. P. Ingalls and G. H. Pettengill, and shown to be comparable in vertical extend to that observed for Venus (about $5 \mathrm{~km}$ ).

\section{Venus}

G. L. Berge, D. O. Muhleman and G. S. Orton report a series of interferometric radiometric observations of Venus at wavelengths of 3,6 and $21 \mathrm{~cm}$, which they have attempted without success to fit to models of the atmosphere and surface derived from probe, radio occultation and (Earthbased) radar data. They conclude that somewhere in the data on which the models are based an error exists. Since no current model can explain the low apparent disk temperatures seen at longer 
wavelengths (e.g. M. Yerbury, D. L. Jauncey and J. C. Condon observe $515 \pm 10 \mathrm{~K}$ at $\lambda=70 \mathrm{~cm}$ ) this failure should, parhaps, not be surprising. W. W. Warnock and J. R. Dickel have observed a disk temperature which is relatively constant at about $700 \mathrm{~K}$ over the wavelength range from 7 to $15 \mathrm{~cm}$. They have attempted to improve the fit of these data to theoretical models of the atmosphere and surface by postulating a two-layer surface, having a low dielectric-constant $(1.5)$ epilith $1 \mathrm{~m}$ thick overlying a much denser core $(\varepsilon=8.5)$. Alternatively, they conclude that there may be an as yet unsuspected significant amount of decimeter-wavelength atmospheric opacity.

Radar observations of Venus have continued to accumulate from which D. B. Campbell et al. have derived a topographic profile along the equator. They find a maximum vertical extent of about $5 \mathrm{~km}$, with a distinct scarp, rising $4 \mathrm{~km}$ in less than $200 \mathrm{~km}$, located near IAU longitude $100^{\circ}$. Improved radar maps of features lying in the hemisphere which is turned towards Earth near inferior conjunction have been obtained by D. B. Campbell $(\lambda=70 \mathrm{~cm}), \mathrm{R}$. M. Goldstein $(\lambda=12 \cdot 5$ $\mathrm{cm})$ and A. E. E. Rogers $(\lambda=3.8 \mathrm{~cm})$.

\section{Mars}

The wealth of new data arising from the various spacecraft orbiting Mars in 1971 and 1972 is too vast to allow for any reasonable summary here. A partial progress report may be gleaned from the references given earlier. Suffice it to say that essentially the whole planet has been mapped from the U.S. Mariner 9 orbiter, in photographs which have a resolution of about a kilometer, and that a smaller number of unusual areas have been observed at resolutions of about a hundred meters. What appear to be volcanic craters complete with lava flows have been found, as well as eroded 'river' beds. Features that on Earth would only be associated with faulting have given rise to the beginnings of a Martian geology. Wind-blown dune fields have been seen with some characteristics that change with time. The polar caps are now thought to consist of several components: a thicker, more permanent layer, probably consisting primarily of water ice, covered with a more transient, thinner layer of carbon dioxide ice. Excellent photographs of Phobos and Deimos were also obtained.

The Martian topography is well established over most of the surface by virtue of observations of ultraviolet scattering and infrared absorption. Confidence has been established by comparison with Earth-based radar observations (now capable of a topographic accuracy of about $100 \mathrm{~m}$ ). The agreement between the former 'pressure' (or density) altitudes and the latter geometric altitudes is excellent over most of the equatorial regions where the latter measurements exist, except in the Tharsis region where a discrepancy of up to $2 \mathrm{~km}$ occurs. This discrepancy is thought to be the result of local gravitational anomalies which have also been detected as perturbations in the spacecraft orbit.

The U.S.S.R. Mars 2 and 3 orbiters carried dual-polarization radiometers which permitted a direct measurement of the surface dielectric constants. Values lying between 2 and 5 have been reported for different areas of the surface, in excellent agreement with the range of values found from Earth-based radar observation. Detailed comparison between the findings of the two methods have not been possible since results for overlapping coverage have not yet been released.

Earth-based observations made during the favorable opposition of 1971, in addition to those obtained by radar, have been reported by several workers. J. Houck et al. have observed a broad absorption band at $2.85 \mu$ from which they infer the presence of minerals on Mars with a bound water content of about $1 \%$. A particle radius of about $100 \mu$ is required by their observations. $\mathrm{T}$. McCord reports successful laboratory modelling of oxidation processes which appear to yield the characteristics observed for the Martian surface.

C. Sagan and J. Veverka have analyzed microwave observations of Mars at many wavelengths and find that there is a significant decrease in the brightness temperature as the wavelength decreases, contrary to what would be expected for a simple surface observed at opposition. They postulate the presence of a non-uniformly distributed layer of liquid water, some tens of microns thick, located within a few millimeters of the surface, as the most likely explanation of the observations.

New data on the pole position of Mars have been obtained from Earth-based observations of Phobos and Deimos by A.T. Sinclair and from an analysis of the position of features seen in 
Mariner 9 photographs. Both sets of results agree remarkably well with values: $(1950 \cdot 0) \alpha=317: 3 \pm$ $\pm 0.2, \delta=52: 7 \pm 0$ : 1 , which differ significantly from those currently adopted by the IAU $\left(\alpha=316^{\circ}: 85\right.$, $\delta=53: 01)$.

\section{CURRENT UNPUBLISHED RESEARCH}

\section{Venus}

T. Gehrels reports that he has noted a secular variation in the UV polarization of the illuminated disk of Venus in the course of his observations 1959-1972. He states, "When Venus is observed near $90^{\circ}$ phase angle, the polarization at $3650 \AA$ is normally several percent positive, due to Rayleigh scattering above the cloudtops. Any day-to-day variation is small ( $<0.5 \%$ polarization). However, a gross depression of the ultraviolet curve by several percent has been observed during 1964-65 and again in 1970-71. The polarization patrol will be continued in an attempt to understand this anomaly. Equivalent photometric patrol observations would be useful.

The global polarization properties of the upper cloud deck are understood in terms of particle shape, refractive index, and sizes. The understanding of regional variations may, however, require polarimetric and photometric observations from a Venus orbiter. Our Venus studies are made by D. L. Coffeen."

\section{Minor Planets}

H. F. Haupt, director of the Graz University Observatory (Austria), reports that their activity has concentrated on photoelectric observations of asteroids, investigating their phase relationship and rotation. He states, "(2) Pallas was observed by H. F. Haupt, A. Schroll, and H. M. Maitzen from 1968 to 1971 at Vienna, Haute Provence (OHP) and ESO yielding final data on its rotational period as well as shape and amplitude of its light-curve. A preliminary report was given at the Bonn meeting of the Astronomische Gesellschaft (Mitt. Astr. Ges. 3, 208, 1972=Mitt. Universitätssternwarte Graz, No. 10). The final publication is hoped to be submitted soon to Icarus.

In summer 1972 H. F. Haupt and H. J. Schober made extensive observations of asteroids No (6) Hebe, (43) Adriadne, and (89) Julia at the Haute Provence Observatory."

\section{Jupiter}

A. Boischot writes that the decametric emission of Jupiter has been studied at Meudon-Nançay since 1970, using the radiospectrograph at 20 to $40 \mathrm{MHz}$. He gives their main results as: "(1) A close relationship is found between the solar wind velocity and the phase of the satellite Io during radio bursts from Jupiter. This is interpreted as resulting from the existence of a plasmasphere around Jupiter. Dynamic models of a plasmasphere have been computed and enable us to explain the part played by Io on the Jupiter decametric emission. (2) We show that the Io phase of the early and the late sources drift by as much as $10^{\circ}$ between 1961 to 1968 . This drift is systematically related to the solar cycle, and also to the jovicentric declination of the Earth. The last three years of data should allow us to distinguish between the two possibilities."

T. Gehrels writes that M. G. Tomasko is computing, using the doubling principle, the intensity emerging from vertically inhomogeneous planetary atmospheres with arbitrary phase functions. One form of this program for Rayleigh scattering has been used to compute the Jovian ultraviolet geometric albedo for various vertical distributions of $\mathrm{NH}_{3}$. Tomasko finds that, while the observed ultraviolet albedo is inconsistent with saturated $\mathrm{NH}_{3}$ down to an $\mathrm{NH}_{3}$ cloud layer, it is consistent with observations to assume reduction of the $\mathrm{NH}_{3}$ saturation to $10 \%$ above the $120 \mathrm{~K}$ level and $100 \%$ below that level. Gehrels also reports that a program for forward scattering phase functions has been written and is being used by $\mathrm{A}$. Clements as part of a $\mathrm{Ph}$. $\mathrm{D}$. dissertation to interpret limb darkening data in the $8900 \AA \mathrm{CH}_{4}$ absorption band. This investigation is expected to yield information on the Jovian vertical cloud structure at two different latitudes. 


\section{Saturn}

G. Godoli writes that observations made at the Catania (Sicily) Astrophysical Observatory of Rhea and Titan in the UBV system show that for Titan a clear dependence on the solar phase angle exists, which has not previously been detected.

\section{IAU PLANETARY PHOTOGRAPHIC DATA CENTER AT MEUDON}

\section{A. Dollfus}

The new IAU Planetary Photographic Data Center of Meudon contains 13 offices, storage facilities for plate collections, a safe for the original negatives, relevant photographic, photometric, optical, micrometric and computing instrumentation, lodging facilities for visitors, and is connected with the $60 \mathrm{~cm}$ and $100 \mathrm{~cm}$ reflectors especially designed for planetary observations.

Dr C. H. Boyer has been appointed curator of the Center.

More than 3000 original negatives are now available for photometric and micrometric work. More than 33000 positive copies on transparent films and 8500 composites are also available for analysis by all qualified scientists wishing to conduct an appropriate research program.

The Data Center has received most of the high-quality planetary plate collections from all parts of the world, from the beginning of astronomical photography until 1968. Since that time the great increase in the number of plates obtained every year by more and more groups of observers, particularly those comprising the Lowell Observatory Planetary Patrol Network, has compelled the replacement of the exhaustive copying and exchange procedures. It was mutually agreed to make available at the Meudon and Lowell Centers only copies of selected plates, and to compile lists of the negatives available for potential users so that they can request photographic copies for special purposes.

The following research projects are among those that have been carried out at the Meudon Center during the past three years using the documents available: Cartography of the surface markings of Mercury based on an analysis of plates taken mainly at the New Mexico State University and Picdu-Midi Observatories and determination of a more accurate period of rotation (J. B. Murray, B. A. Smith and A. Dollfus). Cartography and rotation periods of the four Galilean satellites of Jupiter (J. B. Murray and A. Dollfus). Study of physical behavior of clouds of Venus and general atmospheric circulation (C. Boyer and M. Auriere). A photometric atlas of the isophotes of Venus for different phase angles (Nikander, Bowell and others). An atlas of the surface features of Mars as recorded on photographs taken near opposition since 1907 (G. de Mottoni). Micrometric analysis of the size, shape, and seasonal regression curves for the north polar cap of Mars (A. Dollfus). Synoptic analysis of clouds in Martian atmosphere (Wells). Study of the recurrence of the activity parameters in Jupiter's atmosphere and related radio emissions (R. Prinz and C. Botton).

INTERNATIONAL PLANETARY PATROL PROGRAM

\section{W. A. Baum}

The Planetary Patrol network, managed by the Planetary Research Center at Lowell Observatory, currently includes the Mauna Kea Observatory in Hawaii, the Perth Observatory in Western Australia, the Kavalur Station of the Indian Institute of Astrophysics in southern India, the Republic Observatory station of the South African Astronomical Observatory, the Cerro Tololo InterAmerican Observatory in nothern Chile, and the Lowell Observatory. The Mount Stromlo Observatory in eastern Australia and the Blue Mesa station of New Mexico State University have also participated during part of this program. During favorable periods, uninterrupted photographic surveillance is maintained on Mars, Jupiter, and sometimes Venus. All telescopes of the network are continuously committed and identically equipped. Image sequences are recorded hourly in four colors at a scale of $4.5 / \mathrm{mm}$ on $35-\mathrm{mm}$ film. All films are processed, edited, copied, and catalogued at the Planetary Research Center. 
As of the end of 1972 , the Patrol will have yielded approximately 40800 usable image sequences of Jupiter, 24751 of Mars, and 1540 of Venus. Systematic analyses of these images at the Planetary Research Center are described separately. Access to the patrol films is available to all qualified investigators.

The support of NASA Headquarters is gratefully acknowledged.

PLANETARY RESEARCH CENTER AT THE LOWELL OBSERVATORY

W. A. Baum

The IAU Planetary Research Center at Lowell Observatory has continued to collect and analyze planetary images from all sources. A typical plate or film includes a sequence of planetary images taken in rapid succession in a particular color. As of the end of 1972, the number of catalogued sequences (or composited images) of usable quality from various sources will be approximately as follows:

$\begin{array}{lc}\text { Source } & \text { Image Sequences } \\ \text { International Planetary Patrol } 1969 \rightarrow 1972 & 67000 \\ \text { Lowell Observatory } 1903 \rightarrow 1968 & 10086 \\ \text { Lick Observatory } & 1940 \\ \text { New Mexico State University } & 2052 \\ \text { Planetary Data Center at Meudon } & 1507 \\ \text { Table Mountain Observatory } & 970 \\ \text { Miscellaneous } & 800 \\ & \\ & 84355\end{array}$

The complete facilities of the Center, including access to this collection and the associated machinesearchable IBM card catalogue, are available to all qualified investigators. The International Planetary Patrol Program, which is managed by the Planetary Research Center, is discussed separately.

The research program of the Center includes not only the systematic analysis of photographic images but also the making of photoelectric, spectrophotometric, and polarimetric observations at various telescopes. The regular research staff of the Center includes W. A. Baum (director), P. B. Boyce (astronomer), R. L. Millis (astronomer), L. J. Martin, C. F. Capen, D. T. Thompson, and J. L. Inge. Important responsibilities are carried by H. S. Horstman, S. E. Jones, D. M. Busby, and $J . H$. Chastain, supported by various assistants. A post-doctoral appointment was held by J. J. Caldwell. Other Lowell Observatory staff collaborating in planetary projects include J. S. Hall (director of the Observatory), O. G. Franz (astronomer), and L. A. Riley.

Highlights of recent and current research include a detailed mapping of the developing stages of the 1971 Martian dust storm; a similar study of the 1956 dust storm for comparison; a statistical analysis of the short-term fluctuations in the contrast between neighboring light and dark areas on Mars; the discovery of a diurnal climb of this contrast in some regions; a Mie-scattering interpretation of these results in terms of the rising and settling of Martian dust; the daily mapping of white and yellow Martian clouds so as to determine their statistical distribution and motions; the discovery of a wandering in the apparent outline of Syrtis Major; a spectrophotometric investigation of selected Martian regions; the production of albedo maps of Mars for two seasons and a comparison with the topographical information obtained by Mariner 9; polarimetric photometry of the Jovian Red Spot; a confirmation of the positional wandering of the Red Spot; a comparison of Jovian latitude-rotation profiles of different years; the probable detection of vertical shear in the Jovian atmosphere; photoelectric area-scanning photometry of the inner Saturnian satellites; conventional photoelectric photometry of the intrinsically asymmetric satellite Iapetus; simultaneous two-color photometry of Jovian satellite eclipses; photographic photometry of the occultation of Beta Scorpii by Jupiter for comparison with data for a similar event in 1952; the setting of a limit 
on the transparency of Saturn's outer ring from observations of a stellar occultation; a determination of the 1970 atmospheric circulation period of the Venus atmosphere; experiments with image tremor stabilization; some advances in photoelectric data systems; participation on the Viking (Mars 1975) Orbiter Imaging Team; and participation in the instrument definition phase of the Grand Tour Outer Planets Mission.

The support of NASA Headquarters is gratefully acknowledged.

WORKING GROUP ON MARTIAN NOMENCLATURE

G. de Vaucouleurs

The nomenclature for the 30 Martian provinces has been selected after extensive discussions. These provinces coincide with the 30 charts of the 1:5000000 Mars Atlas in preparation at the U.S. Geological Survey in Flagstaff and at the University of Texas in Austin.

The work on the crater designation is well in hand and should be substantially completed early in January 1973. The present scheme calls for the assigning of names of prominent astronomers, physicists, meteorologists, and other Martian workers to the 120 to 150 largest Martian craters. The survey of the largest Martian craters has been completed and the first list of craters with names is being prepared. For the smaller craters a double letter designation scheme has been adopted which allows for the designation of about 700 craters in each of the 30 provinces. The designation of craters in this scheme should be completed within a few weeks. Dr Carl Sagan has also selected a score of names for the designation of craters on the two Martian satellites. Schemes for the designation of topographic features other than craters, i.e., canyons, valleys, volcanoes, etc. are under active discussion.

G. H. PETTENGILL

President of the Commission 